\title{
Surface waves propagation in fibre-reinforced anisotropic elastic media subjected to gravity field
}

\author{
A. M. Abd-Alla ${ }^{1 \star}$, T. A. Nofal ${ }^{1}$, S. M. Abo-Dahab ${ }^{1,2}$ and A. Al-Mullise ${ }^{1}$ \\ ${ }^{1}$ Mathematics Department, Faculty of Science, Taif University, Saudi Arabia. \\ ${ }^{2}$ Mathematics Department, Faculty of Science, Qena, Egypt, Qena 83523, Egypt.
}

Accepted 28 March, 2013

\begin{abstract}
The objective of this paper is to investigate the surface waves in fibre-reinforced anisotropic elastic medium subjected to gravity field. The theory of generalized surface waves has firstly developed and then it has been employed to investigate particular cases of waves, viz., Stoneley waves, Rayleigh waves and Love waves. The analytical expressions for waves velocity and attenuation coefficient are obtained in the physical domain by using the harmonic vibrations. The wave velocity equations have been obtained in different cases. The numerical results are given and presented graphically. Comparison was made with the results obtained in the presence and absence of gravity and parameters for fibre-reinforced of the material medium. The results indicate that the effect of gravity and parameters of fibre-reinforced of the material medium are very pronounced.
\end{abstract}

Key words: Fibre-reinforced media, surface waves, Stoneley waves, Rayleigh waves, Love waves, gravity.

\section{INTRODUCTION}

The dynamical problem of propagation of surface waves in a homogeneous and non-homogeneous elastic and thermoplastic media are of considerable importance in earthquake, engineering and seismology on account of the occurrence of non-homogeneities in the earth's crust, as the earth is made up of different layers. Abd-Alla et al. (2011) investigated propagation of Rayleigh waves in generalized magneto-thermoelastic orthotropic material under initial stress and gravity field. A Stoneley and Rayleigh wave in a non-homogeneous orthotropic elastic medium under the influence of gravity has been investigated by Abd-Alla and Ahmed (2003). Abd-Alla (1999) studied propagation of Rayleigh waves in an elastic half-space of orthotropic material. Abd-Alla and Ahmed (1999) investigated propagation of Love waves in a non-homogeneous orthotropic elastic layer under initial stress overlying semi-infinite medium. Rayleigh waves in a magnetoelastic half-space of orthotropic material under the influence of initial stress and gravity field investigated by Abd-Alla et al. (2004). Elnaggar and Abd-Alla (1989) studied Rayleigh waves in magneto-thermo-microelastic half-space under initial stress. Abd-Alla and Ahmed (1996) discussed Rayleigh waves in an orthotropic thermoelastic medium under gravity field and initial stress. Propagation of Rayleigh waves in a rotating orthotropic material elastic half-space under initial stress and gravity investigated by Abd-Alla et al. (2012). Wu and Chai (1994) studied propagation of surface waves in anisotropic solids: theoretical calculation and experiment Wu and Liu (1999) investigated the measurement of anisotropic elastic constants of fiber-reinforced composite plate using ultrasonic bulk wave and laser generated Lamb wave.

The group velocity variation of Lamb wave in fiber 
reinforced composite plate studied by Sang-Ho et al. (2007). Fu and Zhang (2006) investigated the continuummechanical modelling of kink-band formation in fibrereinforced composites. Espinosa et al. (2000) discussed the modeling impact induced delamination of woven fiber reinforced composites with contact/cohesive laws. Wave propagation in materials reinforced with bi-directional fibers presented by Weitsman and Benveniste (1974). Weitsman (1972) introduced the wave propagation and energy scattering in materials reinforced by inextensible fibers. Dai and Wang (2006) considered the stress wave propagation in piezoelectric fiber reinforced laminated composites subjected to thermal shock. Tadashi (2000) studied the propagation of Rayleigh waves along an obliquely cut surface in a directional fiber-reinforced composite. Rogerson (1992) investigated the Penetration of impact waves in a six-ply fibre composite laminate. Weitsrian (1992) studied the reflection of harmonic waves in fiber-reinforced materials. Huang et al. (1995) investigated the effect of fibre-matrix interphase on wave propagation along, and scattering from, multilayered fibres in composites.

Transfer matrix approach. Singh and Singh (2004) investigated the reflection of plane waves at the free surface of a fibre-reinforced elastic half-space. Sengupta and Nath (2001) studied the surface waves in fibrereinforced anisotropic elastic media. Sapan and Ranjan (2011) studied the surface wave propagation in fiberreinforced anisotropic elastic layer between liquid saturated porous half space and uniform liquid layer. Chattopadhyay et al. (2002) investigated the reflection of quasi-P and quasi-SV waves at the free and rigid boundaries of a fibre-reinforced medium. Baljeet (2007) discussed the wave propagation in an incompressible transversely isotropic fibre-reinforced elastic media. Baljeet (2005) studied the wave propagation in thermally conducting linear fibre-reinforced composite materials. Abd-Alla et al. (2000) studied the thermal stresses in a non-homogeneous orthotropic elastic multilayered cylinder.

Recently, Abd-Alla and Abo-Dahab (2012) investigated the rotation and initial stress effects on an infinite generalized magneto-thermoelastic diffusion body with a spherical cavity. Abouelregal and Abo-Dahab (2012) discusses the dual phase lag model on magnetothermoelasticity infinite non-homogeneous solid having a spherical cavity.

The present investigation is to study the propagation of surface waves in fibre-reinforced anisotropic elastic medium subjected to gravity field leading to particular cases such as Rayleigh waves, Love waves and Stoneley waves. The waves velocity and attenuation coefficient are obtained in the physical domain by using the harmonic vibrations. The effects of the gravity, anisotropy and parameters for fibre-reinforced of the material medium on surface waves are studied simultaneously.

\section{FORMULATION OF THE PROBLEM}

Let $M_{1}$ and $M_{2}$ be two fires-reinforced elastic anisotropic semi-infinite solid media. They are perfectly welded in contact to prevent any relative motion or sliding before and after the disturbances and that the continuity of displacement, stress etc. hold good across the common boundary surface. Further, the mechanical properties of M1 are different from those of $M_{2}$. These media extend to an infinite great distance from the origin and are separated by a plane horizontal boundary and $M_{2}$ is to be taken above $\mathrm{M}_{1}$.

Let Oxyz be a set of orthogonal Cartesian coordinates and let $\mathrm{O}$ be the any point of the plane boundary and $\mathrm{Oz}$ points vertically downward to the medium $M_{1}$. We consider the possibility of a type of wave travelling in the direction $O x$, in such a manner that the disturbance is largely confined to the neighborhood of the boundary and at any instant, all particles in any line parallel to $y$-axis have equal displacements. These two assumptions conclude that the wave is a surface wave and all partial derivatives with respect to $y$ are zero.

Further let us assume that $u, w$ are the components of displacements at any point $(x, y, z)$ at any time t. It is also assumed that gravitational field produces a hydrostatic initial stress is produced by a slow process of creep where the shearing stresses tend to become smaller or vanish after a long period of time. The equilibrium equation of the initial stress is in the form

$$
\frac{\partial \tau}{\partial x}=0, \frac{\partial \tau}{\partial z}+\rho g=0
$$

The dynamical equations of motion for three-dimensional elastic solid medium under the influence of initial stress and gravity (Sengupta and Nath, 2001) are

$$
\begin{aligned}
& \frac{\partial \tau_{11}}{\partial x}+\frac{\partial \tau_{12}}{\partial y}+\frac{\partial \tau_{18}}{\partial z}+\rho g \frac{\partial w}{\partial x}=\rho \frac{\partial^{x_{u}}}{\partial t^{2}}
\end{aligned}
$$

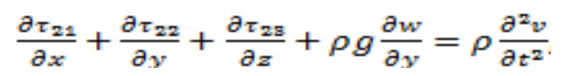

$$
\begin{aligned}
& \frac{\partial \pi_{m B}}{\partial x}+\frac{\partial \tau_{B n}}{\partial y}+\frac{\partial \tau_{B g}}{\partial z}+\rho g \frac{\partial w}{\partial z}=\rho \frac{\partial^{x_{w}}}{\partial t^{2}} . \\
& \text { where, } \frac{\partial w}{\partial z}=-\left(\frac{\partial u}{\partial z}+\frac{\partial v}{\partial y}\right)
\end{aligned}
$$

The Equations (1) becomes

$$
\begin{aligned}
& \frac{\partial \tau_{11}}{\partial x}+\frac{\partial \tau_{18}}{\partial z}+\rho g \frac{\partial w}{\partial x}=\rho \frac{\partial^{2} u}{\partial t^{2}} \\
& \frac{\partial \tau_{21}}{\partial x}+\frac{\partial \tau_{28}}{\partial z}=\rho \frac{\partial^{2} v}{\partial t^{2}}, \\
& \frac{\partial \tau_{s 1}}{\partial x}+\frac{\partial \tau_{z s}}{\partial z}-\rho g \frac{\partial u}{\partial z}=\rho \frac{\partial^{2} w}{\partial t^{2}}
\end{aligned}
$$


where, $\rho$ be the density of the material medium, $g$ be the acceleration due to gravity and $\tau_{i j}=\tau_{j i} \forall(i, j=1,2,3)$ are the stress components.

The constitutive equations for a fibre-reinforced linearly elastic anisotropic medium with respect to a preferred direction $\vec{a}$ are Sapan and Ranjan (2011):

$\tau_{i j}=\lambda e_{k k} \delta_{i j}+2 \mu_{T} e_{i j}+\alpha\left(a_{k} a_{m} e_{k m} \delta_{i j}+e_{k k} a_{i} a_{j}\right)$

$+2\left(\mu_{L}-\mu_{T}\right)\left(a_{i} a_{k} e_{k j}+a_{j} a_{k} e_{k i}\right)+\beta\left(a_{k} a_{m} e_{k m} a_{i} a_{j}\right)$

where, $e_{i j}=\frac{1}{2}\left(u_{i, j}+u_{j, i}\right)$ are components of strain, $\alpha, \beta,\left(\mu_{L}-\mu_{T}\right)$ are reinforced anisotropic elastic parameters, $\quad \mu_{L}, \mu_{T} \quad$ are elastic parameters, $\vec{a}=\left(a_{1}, a_{2}, a_{3}\right)$,

$a_{1}{ }^{2}+a_{2}{ }^{2}+a_{3}{ }^{2}=1$.

If $\vec{a}$ has components that are $(1,0,0)$ so that the preferred direction is the $\mathrm{x}$ - axis, (2) simplifies, as follows

$$
\begin{aligned}
& \tau_{11}=\left(\lambda+2 \alpha+4 \mu_{L}-2 \mu_{T}+\beta\right) \frac{\partial u}{\partial x}+(\lambda+\alpha) \frac{\partial w}{\partial z}, \\
& \tau_{22}=(\lambda+\alpha) \frac{\partial u}{\partial x}+\lambda \frac{\partial w}{\partial z^{3}} \\
& \tau_{33}=(\lambda+\alpha) \frac{\partial u}{\partial x}+\left(\lambda+2 \mu_{T}\right) \frac{\partial w}{\partial z}, \\
& \tau_{12}=\mu_{L} \frac{\partial v}{\partial x}, \\
& \tau_{13}=\mu_{L}\left(\frac{\partial w}{\partial x}+\frac{\partial u}{\partial z}\right), \\
& \tau_{23}=\mu_{T} \frac{\partial v}{\partial z} .
\end{aligned}
$$

By substituting Equations (4) in (2a), (2b) and (2c) it becomes

$\left(\alpha+2 \alpha+4 \mu_{L}-2 \mu_{T}+\beta\right) \frac{\partial^{2} u}{\partial x^{2}}+\left(\alpha+\alpha+\mu_{L}\right) \frac{\partial^{2} W}{\partial x \partial z}+\mu_{L} \frac{\partial^{2} u}{\partial z^{2}}+\rho g \frac{\partial W}{\partial x}=\rho \frac{\partial^{2} u}{\partial t^{2}}$,

$\mu_{L} \frac{\partial^{2} v}{\partial x^{2}}+\mu_{T} \frac{\partial^{2} v}{\partial z^{2}}=\rho \frac{\partial^{2} v}{\partial t^{2}}$

$\mu_{L} \frac{\partial^{x^{2}}}{\partial x^{2}}+\left(\lambda+\alpha+\mu_{L}\right) \frac{\partial^{\mathrm{x}} u}{\partial x \partial z}+\left(\lambda+2 \mu_{T}\right) \frac{\partial^{x^{2} w}}{\partial z^{2}}-\rho g \frac{\partial u}{\partial x}=\rho \frac{\partial^{x^{2} w}}{\partial t^{2}}$.

To examine dilatation and rotational disturbances, we introduce two displacement potentials $\varphi$ and $\psi$ by the Lame's potential method

$u=\frac{\partial \varphi}{\partial x}-\frac{\partial \psi}{\partial z}, \quad w=\frac{\partial \varphi}{\partial z}+\frac{\partial \psi}{\partial x}$.

Now using (8) and substituting in Equations (5) and (7) we obtain the following wave equation for $M_{1}$ satisfied by $\varphi$ and $\psi$ as

$$
\left(\lambda+2 \alpha+4 \mu_{L}-2 \mu_{T}+\beta\right) \frac{\partial^{2} \varphi}{\partial x^{2}}+\left(\lambda+\alpha+2 \mu_{L}\right) \frac{\partial^{2} \varphi}{\partial z^{2}}+\rho g \frac{\partial \psi}{\partial x}=\rho \frac{\partial^{2} \varphi}{\partial t^{2}}
$$

$\left(\alpha+3 \mu_{L}-2 \mu_{T}+\beta\right) \frac{\partial^{2} \psi}{\partial x^{2}}+\mu_{L} \frac{\partial^{\mathrm{x}} \psi}{\partial z^{2}}-\rho g \frac{\partial \varphi}{\partial x}=\rho \frac{\partial^{\mathrm{x}} \psi}{\partial t^{2}}$,

$\mu_{L} \frac{\partial^{2} v}{\partial x^{2}}+\mu_{T} \frac{\partial^{2} v}{\partial z^{2}}=\rho \frac{\partial^{2} v}{\partial t^{2}}$

and similar relations in $M_{2}$ with $\rho, \lambda, \alpha, \mu_{L}, \mu_{T}, \beta$ replaced by $\rho^{\prime}, \lambda_{,}^{\prime}, \alpha^{\prime}, \mu_{L^{3}}^{\prime} \mu^{\prime}{ }_{T}, \beta^{\prime}$

\section{SOLUTION OF THE PROBLEM}

To solve the Equations (9) to (11) assume the following

$\varphi=F(z) e^{i \omega(x-c t)}$

$\psi=G(z) e^{i \omega(x-c t)}$,

$v=H(z) e^{i \omega(x-c t)}$.

Using Equations (12) into (9), (10) and (11) we get a set differential equations for medium $M_{1}$ as follows

$$
\begin{aligned}
& \frac{d^{2} F}{d z^{2}}+h_{1}^{2} F+f_{1}^{2} G=0, \\
& \frac{d^{2} G}{d z^{2}}+l_{1}^{2} G+m_{1}^{2} F=0, \\
& \frac{d^{2} H}{d z^{2}}+k_{1}^{2} H=0
\end{aligned}
$$

Where

$$
\begin{aligned}
& h_{1}^{2}=\frac{\omega^{2}\left(c^{2}-A_{1}\right)}{A_{2}}, \quad f_{1}^{2}=\frac{i \omega g}{A_{2}}, \\
& l_{1}^{2}=\frac{\omega^{2}\left(c^{2}-A_{g}\right)}{A_{4}}, \quad m_{1}^{2}=\frac{i \omega g}{A_{4}}, \\
& k_{1}^{2}=\frac{\omega^{2}\left(c^{2}-A_{4}\right)}{A_{5}}, \\
& A_{1}=\frac{\left(0+2 \alpha+4 \mu_{L}-2 \mu_{T}+\beta\right)}{\rho}, \\
& A_{2}=\frac{\left(\alpha+\alpha+2 \mu_{L}\right)}{\rho}, \\
& A_{3}=\frac{\left(\alpha+3 \mu_{L}-2 \mu_{T}+\beta\right)}{\rho}, \\
& A_{4}=\frac{\mu_{L}}{\rho}, \\
& A_{5}=\frac{\mu_{T}}{\rho} .
\end{aligned}
$$

And the set differential equations for medium $M_{2}$ as follow

$$
\begin{aligned}
& \frac{d^{2} F}{d z^{2}}+h_{1}^{\prime 2} F+f_{1}^{\prime 2} G=0, \\
& \frac{d^{2} G}{d z^{2}}+l_{1}^{\prime 2} G+m_{1}^{\prime 2} F=0, \\
& \frac{d^{2} H}{d z^{2}}+k_{1}^{\prime 2} H=0
\end{aligned}
$$

where 


$$
\begin{aligned}
& h_{1}^{\prime 2}=\frac{\omega^{2}\left(c^{2}-A v_{1}\right)}{A v_{2}}, \quad f_{1}^{\prime 2}=\frac{i \omega g}{A v_{2}{ }^{3}} \\
& l_{1}^{2}=\frac{\omega^{2}\left(c^{2}-A r_{g}\right)}{A v_{4}}, \quad m_{1}^{2}=\frac{i \omega g}{A_{4}}{ }^{x} \\
& k_{1}^{2}=\frac{\omega^{2}\left(c^{2}-A v_{4}\right)}{A_{5}^{t}} \\
& A_{1}=\frac{\left(\nu \nu+2 \alpha^{v}+4 \mu^{r}{ }_{L}-2 \mu^{t}{ }_{T}+\beta \nu\right)}{\rho}, \\
& A_{2}=\frac{\left(\nu+\alpha r+2 \mu r_{L}\right)}{\rho}, \\
& A_{3}=\frac{\begin{array}{c}
\rho \\
\left(\alpha v+3 \mu^{r} L^{-2 \mu^{r}} T_{T}+\beta l\right)
\end{array}}{\rho}, \\
& A_{4}=\frac{\mu \prime_{L}}{\rho}, \\
& A_{5}=\frac{\mu l_{T}}{\rho} \text {. }
\end{aligned}
$$

Equations (13) and (14) must have exponential solutions in order that $\mathrm{F}, \mathrm{G}, \mathrm{H}$ will describe surface waves, they must become vanishingly small as $Z \rightarrow \infty$.

Hence for medium $M_{1}$

$\varphi(x, z, t)=\left[A e^{-P_{1} z}+B e^{-P_{2} z}\right] e^{i \omega(x-c t)}$,

$\psi(x, z, t)=\left[C e^{-P_{1} z}+D e^{-P_{2} z}\right] e^{i \omega(x-c t)}{ }_{1}$

$v(x, z, t)=\left[E e^{-i k_{1} z}\right] e^{i \omega(x-c t)}$

where

$P_{1}=\sqrt{\frac{-a-\sqrt{a^{2}-4 b}}{2}}$,

$P_{2}=\sqrt{-\frac{a}{2}+\frac{1}{2} \sqrt{a^{2}-4 b}}$

$a=\left(l_{1}^{2}+h_{1}^{2}\right), b=\left(h_{1}^{2} l_{1}^{2}-m_{1}^{2} f_{1}^{2}\right)$

and for medium $M_{2}$

$$
\begin{aligned}
& \varphi(x, z, t)=\left[A^{\prime} e^{-P_{1}^{\prime}{ }_{1} z}+B^{\prime} e^{-P^{\prime}{ }_{2} z}\right] e^{i \omega(x-c t)}{ }_{3} \\
& \psi(x, z, t)=\left[C^{\prime} e^{-P_{1}^{\prime}{ }_{1} z}+D^{\prime} e^{-P^{\prime}{ }_{2} z}\right] e^{i \omega(x-c t)}, \\
& v(x, z, t)=\left[E^{\prime} e^{-i k_{1}^{\prime} z}\right] e^{i \omega(x-c t)}
\end{aligned}
$$

where

$$
\begin{aligned}
& P_{1}^{\prime}=\sqrt{\frac{-a-\sqrt{a^{2}-4 b^{\prime}}}{2}}, \\
& P_{2}^{\prime}=\sqrt{-\frac{a^{\prime}}{2}+\frac{1}{2} \sqrt{a^{\prime 2}-4 b^{\prime}}}, \\
& a^{\prime}=\left(l_{1}^{\prime \prime 2}+h_{1}^{\prime 2}\right), b^{\prime}=\left(h_{1}^{\prime 2} l_{1}^{\prime 2}-m_{1}^{\prime 2} f_{1}^{\prime 2}\right) .
\end{aligned}
$$

To reduce the constants in the equations (15), (16) to be 5 instead of 10 constants, we follow the following

$$
\begin{aligned}
& C=\gamma_{1} A, \quad D=\gamma_{2} B_{3} \\
& C^{\prime}=\gamma_{1}^{\prime} A^{\prime}, \quad D^{\prime}=\gamma_{2}^{\prime} B^{\prime}
\end{aligned}
$$

where, $\gamma_{j}=\frac{m_{1}^{2}}{p_{i}^{2}+l_{1}^{2}}, \gamma_{j}^{\prime}=\frac{m v_{1}^{2}}{p_{i}^{2}+v_{1}^{2}}, i, j=1,2$. After solving equations (15) and (16) we are substituting the values of $\varphi$ and $\psi$ into equations (8), produced from the values of into $u, w, u^{\prime}$ and $w^{\prime}$ which are as follows

$$
\begin{gathered}
u=\left[\left(i \omega+\gamma_{1} P_{1}\right) A e^{-P_{1} z}+\left(i \omega+\gamma_{2} P_{2}\right) B e^{-P_{2} z}\right] e^{i \omega(x-c t)}, \\
w=\left[\left(i \omega \gamma_{1}-P_{1}\right) A e^{-P_{1} x}+\left(i \omega \gamma_{2}-P_{2}\right) B e^{-P_{2} z}\right] e^{i \omega(x-c t)}, \\
u^{\prime}=\left[\left(i \omega+\gamma_{1}^{\prime} P_{1}^{\prime}\right) A^{\prime} e^{-P_{1}^{\prime} x}+\left(i \omega+\gamma_{2}^{\prime} P_{2}^{\prime}\right) B^{\prime} e^{-P_{2}^{\prime} z}\right] e^{i \omega(x-c t)}, \\
w^{\prime}=\left[\left(i \omega \gamma_{1}^{\prime}-P_{1}^{\prime}\right) A^{\prime} e^{-P_{1}^{\prime} z}+\left(i \omega \gamma_{2}^{\prime}-P_{2}^{\prime}\right) B^{\prime} e^{-P_{2}^{\prime} z}\right] e^{i \omega(x-c t)} .
\end{gathered}
$$

\section{BOUNDARY CONDITIONS}

The boundary conditions in the problem are:

(i) The displacement components at the boundary surface between the media $M_{1}$ and $M_{2}$ must be continuous at all times and positions. This means that:

$[u, v, w] M_{1}=[u, v, w] M_{2}$ at $\mathrm{z}=0$

then we obtain

$\frac{\partial \varphi}{\partial x}-\frac{\partial \psi}{\partial z}=\frac{\partial \varphi^{v}}{\partial x}-\frac{\partial \psi}{\partial z}$.

$E e^{-i k_{1} z}=E^{\prime} e^{-i k v_{1} z}$

$\frac{\partial \varphi}{\partial z}+\frac{\partial \psi}{\partial x}=\frac{\partial \varphi^{v}}{\partial z}+\frac{\partial \psi^{\prime}}{\partial x}$.

$\left(1-i \gamma_{1} \beta_{1}\right) A+\left(1-i \gamma_{2} \beta_{2}\right) B-\left(1-i \gamma_{1}^{s} \beta_{1}^{s}\right) A^{s}-\left(1-i \gamma_{2}^{s} \beta_{2}^{s}\right) B^{s}=0$,

$E-E^{\prime}=0$,

$\left(\gamma_{1}+i \beta_{1}\right) A+\left(\gamma_{2}+i \beta_{2}\right) B-\left(\gamma_{1}^{s}+i \beta_{1}^{r}\right) A^{\prime}-\left(\gamma_{2}^{s}+i \beta_{2}^{r}\right) B^{\prime}=0$.

(ii) The stress components $\tau_{13}, \tau_{23}$ and $\tau_{33}$ must be continuous at the boundary $z=0$

$\left[\tau_{13}, \tau_{23}, \tau_{33}\right] M_{1}=\left[\tau_{13}, \tau_{23}, \tau_{33}\right] M_{2}$, at $\mathrm{z}=0$

$\tau_{13}=\tau_{13^{\prime}}^{\prime}$

$\mu_{L}\left(\frac{\partial w}{\partial x}+\frac{\partial u}{\partial z}\right)=\mu_{L}^{\prime}\left(\frac{\partial w^{\prime}}{\partial x}+\frac{\partial u^{\prime}}{\partial z}\right)$

$\mu_{L}\left[\left(-\omega^{2} \gamma_{1}-2 i \omega P_{1}-\gamma_{1} P_{1}^{2}\right) A+\left(-\omega^{2} \gamma_{2}-2 i \omega P_{2}-\gamma_{2} P_{2}^{2}\right) B\right]$

$+\mu_{L}^{\prime}\left[\left(\omega^{2} Y_{1}^{\prime}+2 i \omega P_{1}^{\prime}+\gamma_{1}^{\prime} P_{1}^{\prime}{ }_{1}^{2}\right) A^{\prime}+\left(\omega^{2} \gamma_{2}^{\prime}+2 i \omega P_{2}^{\prime}+\gamma_{2}^{\prime} P_{2}^{\prime}{ }_{2}^{2}\right) B^{\prime}=0\right.$.

$\tau_{23}=\tau_{23^{\prime}}^{\prime}$

$\mu_{T} \frac{\partial v}{\partial z}=\mu_{T}^{\prime} \frac{\partial v^{\prime}}{\partial z^{\prime}}$

$i k_{1} \mu_{T} E-i k_{1}^{\prime} \mu_{T}^{\prime} E^{\prime}=0$, 


$$
\begin{aligned}
& E-E^{\prime}=0 \text {, } \\
& \tau_{33}=\tau_{33}^{\prime} \text {, } \\
& (\lambda+\alpha) \frac{\partial u}{\partial x}+\left(\lambda+2 \mu_{T}\right) \frac{\partial w}{\partial z}=\left(\lambda^{\prime}+\alpha^{\prime}\right) \frac{\partial u^{\prime}}{\partial x}+\left(\lambda^{\prime}+2 \mu^{\prime}\right) \frac{\partial w^{\prime}}{\partial z}, \\
& {\left[\lambda\left(\beta_{1}^{2}-1\right)+a\left(i \gamma_{1} \beta_{1}-1\right)+2 \mu_{T}\left(\beta_{1}{ }^{2}-i \gamma_{1} \beta_{1}\right)\right]_{A}} \\
& +\left[\lambda\left(\beta_{2}{ }^{2}-1\right)+\alpha\left(i \gamma_{2} \beta_{2}-1\right)+2 \mu_{T}\left(\beta_{2}{ }^{2}-i \gamma_{2} \beta_{2}\right)\right]_{B} \\
& -\left[\lambda^{\prime}\left(\beta_{1}^{\prime}{ }_{1}^{2}-1\right)+\alpha^{t}\left(i \gamma_{1}^{\prime} \beta_{1}^{\prime}-1\right)+2 \mu_{T}^{\prime}\left(\beta_{1}^{\prime}{ }_{1}^{2}-i \gamma_{1}^{\prime} \beta_{1}^{\prime}\right)\right] A^{\prime} \\
& -\left[\lambda^{\prime}\left(\beta^{\prime}{ }_{2}{ }^{2}-1\right)+a^{\prime}\left(i \gamma^{\prime}{ }_{2} \beta^{\prime}{ }_{2}-1\right)+2 \mu_{T}^{\prime}\left(\beta^{\prime}{ }_{2}{ }^{2}-i \gamma^{\prime}{ }_{2} \beta_{2}{ }_{2}\right)\right] B^{\prime}=0
\end{aligned}
$$

Where

$$
\begin{aligned}
& \beta_{j}=\frac{P_{i}}{\omega}, i=j=1,2, \\
& \beta^{\prime}{ }_{j}=\frac{P_{i}}{\omega}, i=j=1,2 .
\end{aligned}
$$

Eliminating the constants $A, B, E, A^{\prime}, B^{\prime}$ and $E^{\prime}$ from Equations (18) to (23) we get

$$
\operatorname{Det}\left(a_{i j}\right)=0, \quad \tilde{i}_{a j}=1,2,3,4,5,6
$$

where

$$
\begin{aligned}
& a_{11}=1-i \gamma_{2} \beta_{1}, a_{12}=1-i \gamma_{2} \beta_{2}, a_{13}=0, a_{14}=-\left(1-i \gamma_{2}^{0} \beta_{1}^{p}\right) \text {, } \\
& a_{15}=-\left(1-i \gamma_{2} \beta_{2}^{s}\right), a_{16}=0 \text {, } \\
& a_{21}=\gamma_{1}+i \beta_{1} \quad, \quad a_{22}=\gamma_{2}+i \beta_{2} \quad, \quad a_{2 a}=0 \quad \text {, } \\
& a_{24}=-\left(\gamma_{1}^{g}+i \beta_{1}^{g}\right), a_{25}=-\left(\gamma_{2}^{r}+i \beta_{2}^{g}\right), a_{26}=0 \text {, } \\
& a_{31}=0, a_{32}=0, a_{33}=1, a_{34}=0, a_{35}=0, a_{36}=-1 \text {, } \\
& a_{41}=\mu_{1}\left(-\omega^{2} \gamma_{1}-2 i \omega P_{1}-\gamma_{1} P_{1}^{2}\right) \\
& a_{42}=\mu_{1}\left(-\omega^{2} \gamma_{2}-2 i \omega P_{2}-\gamma_{2} P_{2}^{2}\right), a_{43}=0, \\
& a_{44}=\mu_{1}^{\circ}\left(\omega^{2} \gamma_{2}^{0}+2 i \omega P_{2}^{0}+\gamma_{2}^{0} P_{2}^{0}\right) \\
& a_{45}=\mu_{1}^{\circ}\left(\omega^{2} \gamma_{2}^{\circ}+2 i \omega P_{2}^{0}+\gamma_{2}^{\circ} P_{2}^{0}\right), a_{46}=0 \text {, } \\
& a_{51}=0, a_{52}=0, a_{53}=i k_{1} \mu_{T}, a_{54}=0, a_{55}=0 \text {, } \\
& a_{56}=-i k_{1}^{t} \mu_{T^{\prime}}^{\prime} \\
& a_{61}=2\left(\beta_{1}^{2}-1\right)+\alpha\left(i \gamma_{2} \beta_{1}-1\right)+2 \mu_{2}\left(\beta_{1}^{2}-\mu_{1} \beta_{1}\right) \text {, } \\
& a_{62}=2\left(\beta_{2}^{2}-1\right)+\alpha\left(i \gamma_{2} \beta_{2}-1\right)+2 \mu_{2}\left(\beta_{2}^{2}-4_{2} \beta_{2}\right) \text {, } \\
& a_{63}=0 \text {. }
\end{aligned}
$$

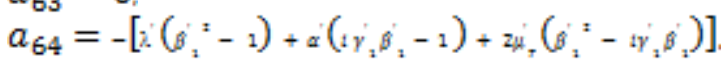

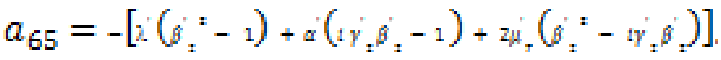

$$
\begin{aligned}
& a_{66}=0 \text {. }
\end{aligned}
$$

From Equation (24), we get the velocity of surface waves in common boundary between two fibre-reinforced elastic anisotropic semi-infinite solid media under the influence of gravity, since the wave velocity c obtained from (24) depends on the particular value of $\omega$ which indicates to the dispersion of the general wave form and on the gravity field, imposing a certain changes in the waves form.

\section{PATICULAR CASES}

\section{Stoneley waves}

It is the generalized form of Rayleigh waves in which we assume that the waves are propagated along the common boundary of two semi-infinite media $M_{1}$ and $M_{2}$. Therefore, Equation (24) determines the wave velocity equation for Stoneley waves in anisotropic fibrereinforced solid elastic media under the influence of gravity.

Clearly from Equation (24), it is follows that wave velocity of the Stoneley waves depends upon the parameters for fibre-reinforced of the material medium, gravity and the densities of both media. Since the wave velocity Equation (24) for Stoneley waves under the presence circumstances depends on the particular value of $\omega$ and creates a dispersion of a general wave form.

Further, Equation (24), of course, is in complete agreement with the corresponding classical result, when the effect of gravity and parameters of the fibre-reinforcement are ignored.

\section{Rayleigh waves}

To investigate the possibility of Rayleigh waves in anisotropic fibrereinforced elastic media, we replace medium $M_{2}$ by a vacuum, in the preceeding problem. Since the boundary $z=0$ is adjacent to vacuum, it is free from surface traction. So the stress boundary condition in this case may be expressed as:

$\tau_{31}=\tau_{33}=0$ at $z=0$,

$\tau_{31}=0$ at $z=0$,

which reduces to

$\mu_{L}\left(\frac{\partial w}{\partial x}+\frac{\partial u}{\partial z}\right)=0$,

$\left(\omega^{2} \gamma_{1}+2 i \omega P_{1}+\gamma_{1} P_{1}^{2}\right) A+\left(\omega^{2} \gamma_{2}+2 i \omega P_{2}+\gamma_{2} P_{2}^{2}\right) B=0$,

$\tau_{\mathrm{aa}}=0$ at $z=0$

which tends to

$$
\begin{aligned}
& (\lambda+\alpha) \frac{\partial u}{\partial x}+\left(\lambda+2 \mu_{T}\right) \frac{\partial w}{\partial z}=0, \\
& \begin{aligned}
{\left[\lambda\left(\beta_{1}{ }^{2}-2 i \gamma_{1} \beta_{1}-1\right)-\alpha\left(i \gamma_{1} \beta_{1}+1\right)+2 \mu_{T}\left(\beta_{1}{ }^{2}-i \gamma_{1} \beta_{1}\right)\right]_{A} } \\
+\left[\lambda\left(\beta_{2}{ }^{2}-2 i \gamma_{2} \beta_{2}-1\right)-\alpha\left(i \gamma_{2} \beta_{2}+1\right)+2 \mu_{T}\left(\beta_{2}{ }^{2}-i \gamma_{2} \beta_{2}\right)\right] B=0 .
\end{aligned}
\end{aligned}
$$

The frequency equation for Rayleigh waves in isotropic elastic medium given in the following form;

$\operatorname{Det}\left(a_{i j}\right)=0, \quad \bar{i}_{i}, j=1,2$

where

$a_{11}=\omega^{2} \gamma_{1}+2 i \omega P_{1}+\gamma_{1} P_{1}^{2}$,

$a_{12}=\omega^{2} \gamma_{2}+2 i \omega P_{2}+\gamma_{2} P_{2}^{2}$.

$a_{21}=\lambda\left(\beta_{1}{ }^{2}-2 i \gamma_{1} \beta_{1}-1\right)-\alpha\left(i \gamma_{1} \beta_{1}+1\right)+2 \mu_{7}\left(\beta_{1}{ }^{2}-i \gamma_{1} \beta_{1}\right)$.

$a_{22}=\lambda\left(\beta_{2}{ }^{2}-2 i \gamma_{2} \beta_{2}-1\right)-\alpha\left(i \gamma_{2} \beta_{2}+1\right)+2 \mu_{T}\left(\beta_{2}{ }^{2}-i \gamma_{2} \beta_{2}\right)$.

\section{Love waves}

To investigate the possibility of Love waves in a fibre-reinforced elastic solid media, we replace medium $M_{2}$ is obtained by two horizontal plane surface at a distance $\mathrm{H}$-apart, while $M_{1}$ remains infinite.

For medium $M_{1}$, the displacement component $v$ remains same as in general case given by Equation (15). For the medium $M_{2}$, we preserve the full solution, since the displacement component along $y$-axis that is, $v$ no longer diminishes with increasing distance from the boundary surface of two media. 
In this case the boundary conditions are

$$
\begin{aligned}
& \tau_{13}=0, \tau_{\mathrm{ga}}=0, v=v^{\prime} \text { at } z=0 \text {, }
\end{aligned}
$$

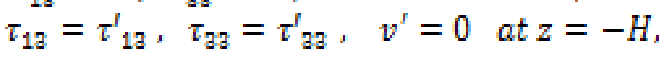

$$
\begin{aligned}
& \tau_{13}=0 \quad \text { at } z=0 \text {. } \\
& \mu_{L}\left(\frac{\partial w}{\partial x}+\frac{\partial u}{\partial z}\right)=0 \text {, } \\
& \left(\omega^{2} \gamma_{1}+2 i \omega P_{1}+\gamma_{1} P_{1}^{2}\right) A+\left(\omega^{2} \gamma_{2}+2 i \omega P_{2}+\gamma_{2} P_{2}^{2}\right) B=0 \text {. } \\
& \tau_{\mathrm{ag}}=0 \text { at } z=0 \text {, } \\
& (\lambda+\alpha) \frac{\partial u}{\partial x}+\left(\alpha+2 \mu_{T}\right) \frac{\partial w}{\partial z}=0 \text {, } \\
& {\left[\lambda\left(P_{1}^{2}-\omega^{2}\right)+a\left(i \omega \gamma_{1} P_{1}-\omega^{2}\right)+2 \mu_{T}\left(P_{1}^{2}-i \omega \gamma_{1} P_{1}\right)\right] A} \\
& +\left[\lambda\left(P_{2}^{2}-\omega^{2}\right)+a\left(i \omega \gamma_{2} P_{2}-\omega^{2}\right)+2 \mu_{T}\left(P_{2}^{2}-i \omega \gamma_{2} P_{2}\right)\right] B=0 \\
& v=v^{\prime} \quad \text { at } z=0 \\
& E-E^{\prime}=0 \text {, } \\
& \tau_{19}=\tau_{13}^{r} \text { at } z=-H_{1} \\
& \mu_{L}\left(\frac{\partial w}{\partial x}+\frac{\partial u}{\partial z}\right)=\mu_{L}^{\prime}\left(\frac{\partial w^{f}}{\partial x}+\frac{\partial u^{f}}{\partial z}\right)
\end{aligned}
$$

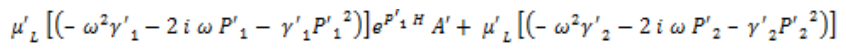

$$
\begin{aligned}
& \mathrm{X} e^{P_{2}^{\prime}{ }^{H}} B^{y}=0 \\
& \tau_{\mathrm{ga}}=\tau_{\mathrm{aa}}^{t} \quad \text { at } z=-H_{\text {, }} \\
& (a+\alpha) \frac{\partial u}{\partial x}+\left(\alpha+2 \mu_{T}\right) \frac{\partial w}{\partial z}=\left(\alpha^{s}+\alpha^{r}\right) \frac{\partial u^{r}}{\partial x}+\left(\lambda+2 \mu_{T}^{s}\right) \frac{\partial w^{s}}{\partial z} . \\
& {\left[\gamma\left(P_{1}^{2}-\omega^{2}\right)+\alpha\left(i \omega \gamma_{1} P_{1}-\omega^{2}\right)+2 \mu_{T}\left(P_{1}^{2}-i \omega \gamma_{1} P_{1}\right)\right] e^{P_{1} H} A+\left[\lambda\left(P_{2}^{2}-\omega^{2}\right)+\right.} \\
& \left.\alpha\left(i \omega \gamma_{2} P_{2}-\omega^{2}\right)+2 \mu_{T}\left(P_{2}{ }^{2}-i \omega \gamma_{2} P_{2}\right)\right] e^{P_{2} H} B-\left[\lambda^{\prime}\left(P^{\prime}{ }_{1}{ }^{2}-\omega^{2}\right)+\alpha^{\prime}\left(i \omega \gamma_{1}{ }_{1}^{\prime} P_{1}^{\prime}-\right.\right. \\
& \left.\left.\omega^{2}\right)+2 \mu_{T}^{\prime}\left(P_{1}^{\prime 2}-i \omega \gamma_{1}^{\prime} P_{1}^{\prime}\right)\right] e^{P^{\prime}{ }_{1}{ }^{\prime}} A^{\prime}-\left[\lambda^{\prime}\left(P_{2}^{\prime}{ }_{2}^{2}-\omega^{2}\right)+\alpha^{\prime}\left(i \omega \gamma_{2}^{\prime} P_{2}^{\prime}{ }_{2}-\omega^{2}\right)+\right. \\
& \left.2 \mu_{T}^{\prime}\left(P_{2}^{\prime}{ }_{2}{ }^{2}-i \omega y_{2}^{\prime} P_{2}^{\prime}{ }_{2}\right)\right] e^{P_{2}^{\prime}{ }_{2} B^{\prime}}=0 \text { 。 } \\
& v^{\prime}=0 \text { at } z=-H \text {, } \\
& E^{\prime}=0 \text {. }
\end{aligned}
$$

In this case the velocity wave it has given from the following equation

$\operatorname{Det}\left(a_{i j}\right)=0, \quad i_{i} j=1,2,3,4,5,6$

where:

$$
\begin{aligned}
& a_{11}=\left(\omega^{2} \gamma_{1}+2 i \omega P_{1}+\gamma_{1} P_{1}^{2}\right), \\
& a_{12}=\left(\omega^{2} \gamma_{2}+2 i \omega P_{2}+\gamma_{2} P_{2}^{2}\right), \\
& a_{13}=0, \quad a_{14}=0, \quad a_{15}=0, \quad a_{16}=0, \\
& a_{21}=\left[\lambda\left(P_{1}^{2}-\omega^{2}\right)+\alpha\left(i \omega \gamma_{1} P_{1}-\omega^{2}\right)+2 \mu_{T}\left(P_{1}^{2}-i \omega \gamma_{1} P_{1}\right)\right], \\
& a_{22}=\left[\lambda\left(P_{2}^{2}-\omega^{2}\right)+\alpha\left(i \omega \gamma_{2} P_{2}-\omega^{2}\right)+2 \mu_{T}\left(P_{2}^{2}-i \omega \gamma_{2} P_{2}\right)\right], \\
& a_{23}=0, \quad a_{24}=0, \quad a_{25}=0, \quad a_{26}=0, \\
& a_{31}=0, a_{22}=0, a_{3 a}=1, a_{34}=0, a_{25}=0, a_{36}=-1, \\
& a_{41}=\mu_{L}\left[\left(-\omega^{2} \gamma_{1}-2 i \omega P_{1}-\gamma_{1} P_{1}^{2}\right)\right] e^{P_{1} H}{ }_{x} \\
& a_{42}=\mu_{L}\left[\left(-\omega^{2} \gamma_{2}-2 i \omega P_{2}-\gamma_{2} P_{2}{ }^{2}\right)\right] e^{P_{2} H}, \\
& a_{43}=0,
\end{aligned}
$$

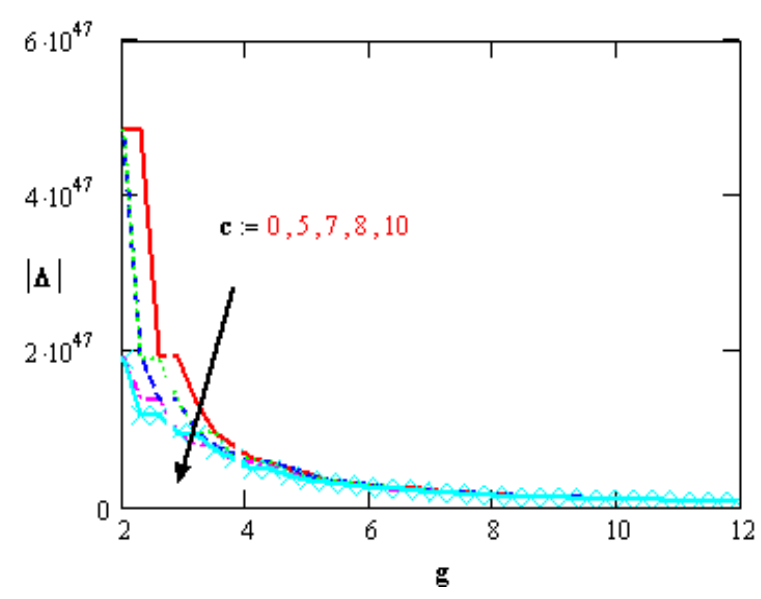

Figure 1. Variation of the secular equation with varies values of $c$ with respect to gravity $g$.

$$
\begin{aligned}
& a_{44}=\mu_{L}^{s}\left[\left(-\omega^{2} \gamma_{1}^{s}-2 i \omega P_{1}^{\prime}-\gamma_{1}^{s}{ }_{1} P_{1}^{\prime}{ }_{1}^{2}\right)\right] e^{P_{1}^{\prime} H^{\prime}} \\
& a_{45}=\mu_{L}^{s}\left[\left(-\omega^{2} \gamma_{2}^{s}-2 i \omega P_{2}^{\prime}-\gamma_{2}^{s} P_{2}^{\prime}\right)\right] e^{P_{2}^{\prime}}{ }_{2} \text {, } \\
& a_{46}=0 \text {, } \\
& a_{51}=\left[\lambda\left(P_{1}^{2}-\omega^{2}\right)+\alpha\left(i \omega \gamma_{1} P_{1}-\omega^{2}\right)+2 \mu_{T}\left(P_{1}^{2}-i \omega \gamma_{1} P_{1}\right)\right] e^{P_{1} H} \\
& a_{52}=\left[\lambda\left(P_{2}{ }^{2}-\omega^{2}\right)+\alpha\left(i \omega \gamma_{2} P_{2}-\omega^{2}\right)+2 \mu_{T}\left(P_{2}{ }^{2}-i \omega \gamma_{2} P_{2}\right)\right] e^{P_{2} H}, \\
& a_{5 a}=0 \text {, } \\
& a_{54}=-\left[\lambda^{\prime}\left(P_{1}^{\prime}{ }_{1}^{2}-\omega^{2}\right)+\alpha^{\prime}\left(i \omega y_{1}^{\prime} P_{1}^{\prime}-\omega^{2}\right)+2 \mu_{T}^{\prime}\left(P_{1}^{\prime}{ }_{1}^{2}-i \omega \gamma_{1}^{\prime} P_{1}^{\prime}\right)\right] e^{p^{\prime}{ }_{1}{ }^{H}}
\end{aligned}
$$

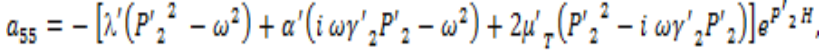

$$
\begin{aligned}
& a_{56}=0 \text {, } \\
& a_{61}=0, \quad a_{62}=0, \quad a_{61}=0, \quad a_{64}=0, \quad a_{65}=0, \quad a_{66}=1 .
\end{aligned}
$$

\section{NUMERICAL RESULTS AND DISCUSSION}

The following values of elastic constants are considered (Chattopadhyay et al., 2002; Singh and Singh, 2004), for mediums $M_{1}$ and $M_{2}$ respectively.

$\rho=2660 \mathrm{Kg} / \mathrm{m}^{3}, \lambda=5.65 \times 10^{10} \mathrm{Nm}^{-2}, \mu_{T}=2.46 \times 10^{9} \mathrm{Nm}^{-2}, \mu_{L}=5.66 \times 10^{9} \mathrm{Nm}^{-2}$, $\alpha=-1.28 \times 10^{9} \mathrm{Nm}^{-2}, \beta=220.90 \times 10^{9} \mathrm{Nm}^{-2}$,

$\rho=7800 \mathrm{Kg} / \mathrm{m}^{3}, \lambda=5.65 \times 10^{9} \mathrm{Nm}^{-2}, \mu_{T}=2.46 \times 10^{10} \mathrm{Nm}^{-2}, \mu_{L}=5.66 \times 10^{10} \mathrm{Nm}^{-2}$, $\alpha=-1.28 \times 10^{10} \mathrm{Nm}^{-2}, \beta=220.90 \times 10^{10} \mathrm{Nm}^{-2}$

$c_{v}=0.787 \times 10^{3} \mathrm{~J} / \mathrm{kg} \mathrm{K}, K=0.0921 \times 10^{3} \mathrm{Jm}^{-1} \mathrm{deg}^{-1} \mathrm{~s}^{-1}, T_{0}=293 \mathrm{~K}, \mathrm{c}=1.2 \times 10^{4} \mathrm{~m}^{2} / \mathrm{s}^{2} \mathrm{~K}$.

The numerical technique outlined above was used to obtain surface wave velocity and with respect to wave number under the effects of gravity and thermal relaxation time parameter in two models. For the sake of brevity some computational results are being presented here. The variations are shown in Figures 1 to 13 respectively.

Figure 1 shows the variation of the secular equation of surface waves, which it decreases with increasing of gravity field until approaching to zero, as well it decreases with increasing of the value of wave speed.

Figure 2 shows the variation of Stoneley wave velocity 

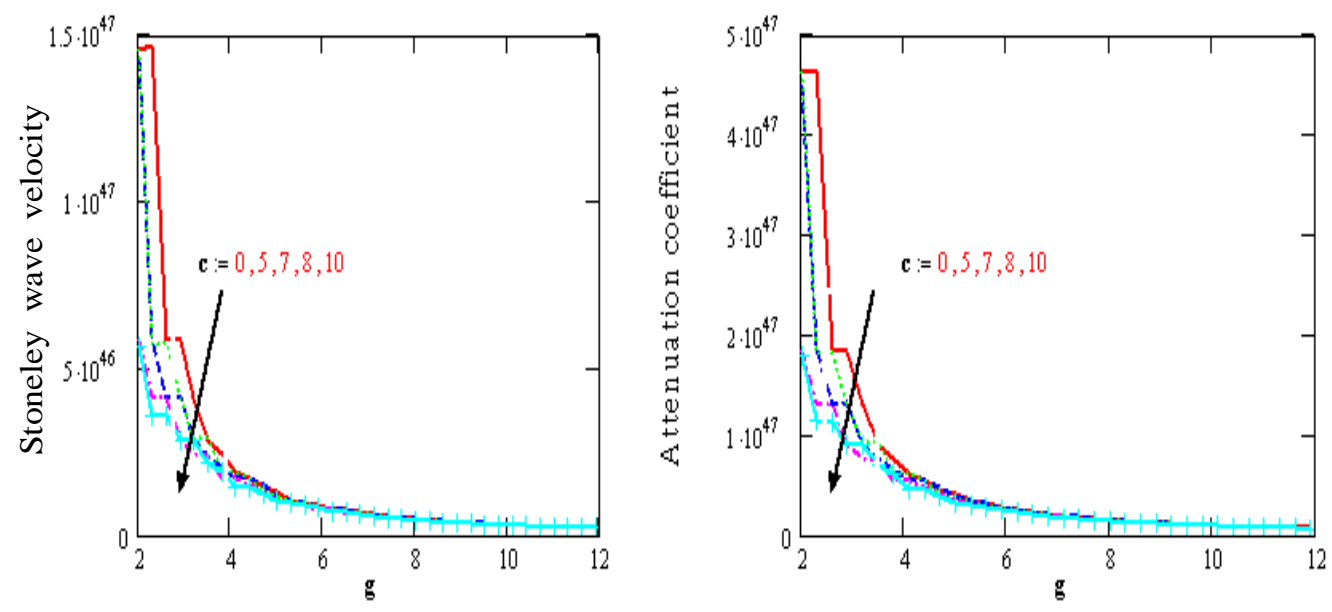

Figure 2. Variation of the Stoneley wave velocity and attenuation coefficient with varies values of $c$ with respect to gravity $\mathrm{g}$.

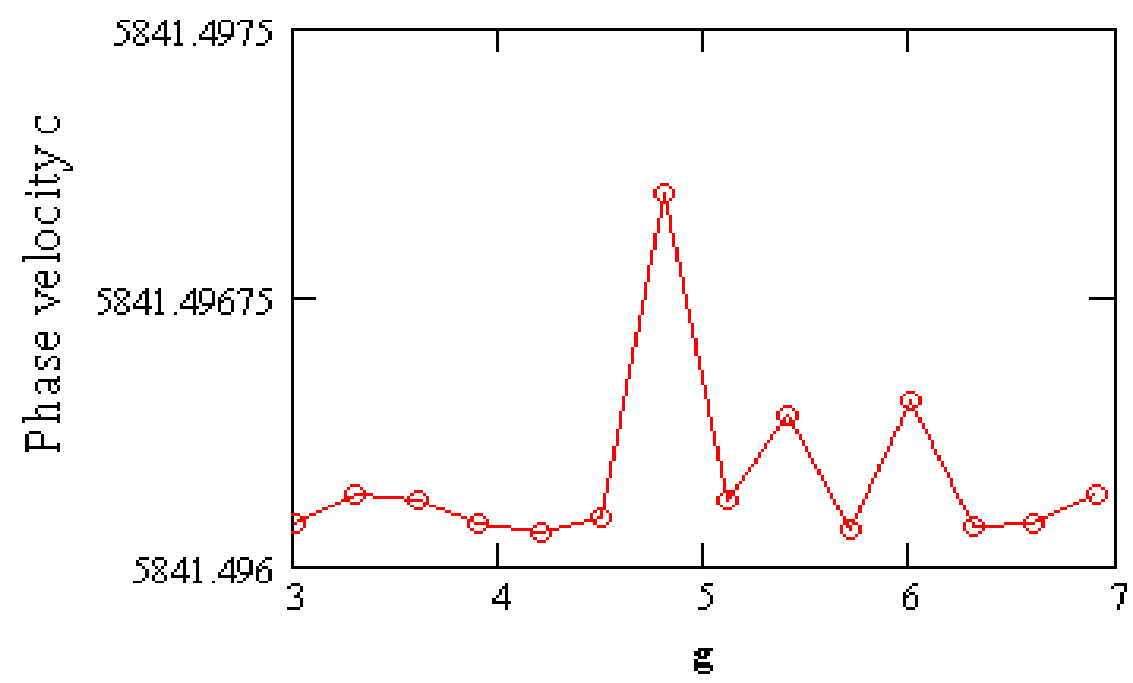

Figure 3. Phase velocity $\mathrm{c}$ with respect to gravity $\mathrm{g}$.

and attenuation coefficient of surface waves under the effects of gravity field. The effect of gravity on Stoneley wave velocity which it decreases with increasing of gravity field, as well it decreases with increasing of the value of wave speed. Figure 3 shows the variation of phase velocity of surface waves under the effects of gravity. The value of the phase velocity has an oscillatory behavior with gravity in the whole range of the gravity field. Figure 4 show the variation of Stoneley wave velocity and attenuation coefficient of surface waves under the effect of gravity. The effect of gravity $g$ on Stoneley wave velocity which it decreases with increasing of gravity field, as well it decreases with increasing of the value of wave speed. At a given instant, the velocity of Stoneley waves is finite, which is due to the effect of gravity. Figure 5 show the the variation secular equation of surface waves under the effect of gravity. The effect of gravity $g$ on secular equation which it decreases with increasing of gravity field, as well it decreases with increasing of the value of wave speed. Figures 6 and 8 show the the variation secular equation for Rayleigh wave under the effect of gravity. The effect of gravity $g$ on secular equation which it decreases with increasing of gravity field, as well it decreases with increasing of the value of wave speed. Figure 7 shows that the variation of phase velocity of Rayleigh wave under the effects of gravity. The value of the phase velocity has an oscillatory behavior with gravity in the whole range of the gravity field. Figures 9 and 10 show the variation of Love wave velocity with respect to depth $\mathrm{H}$, for different values of 


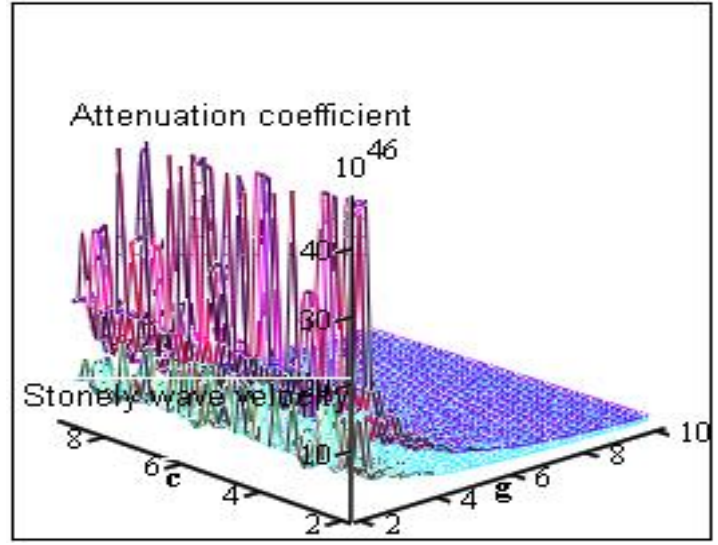

Figure 4. Variation of the Rayleigh wave velocity and attenuation coefficient with varies values of $g$ and $c$.

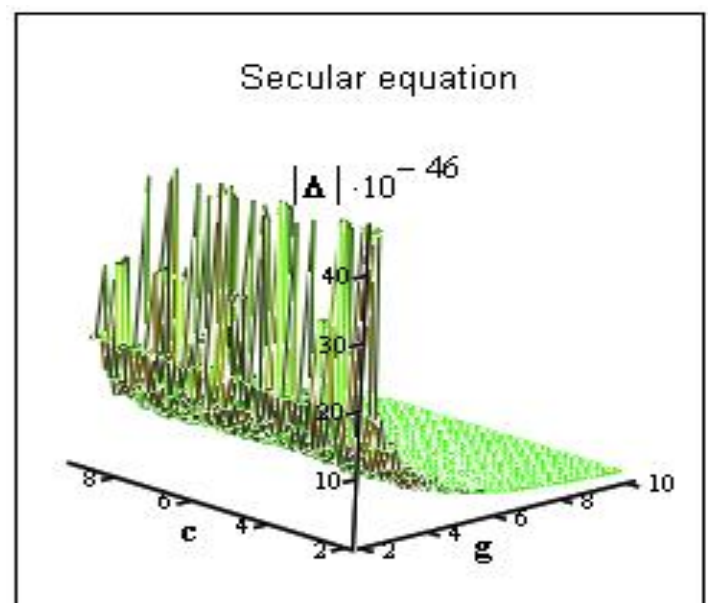

Figure 5. Variation of the secular equation with varies values of $g$ and $c$.

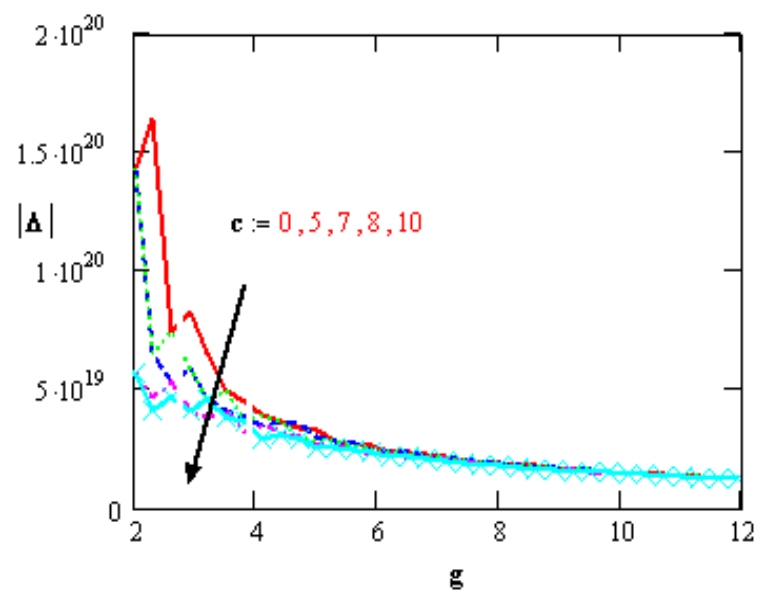

Figure 6. Variation of the secular equation for Rayleigh wave with varies values of $c$ with respect to gravity $g$.

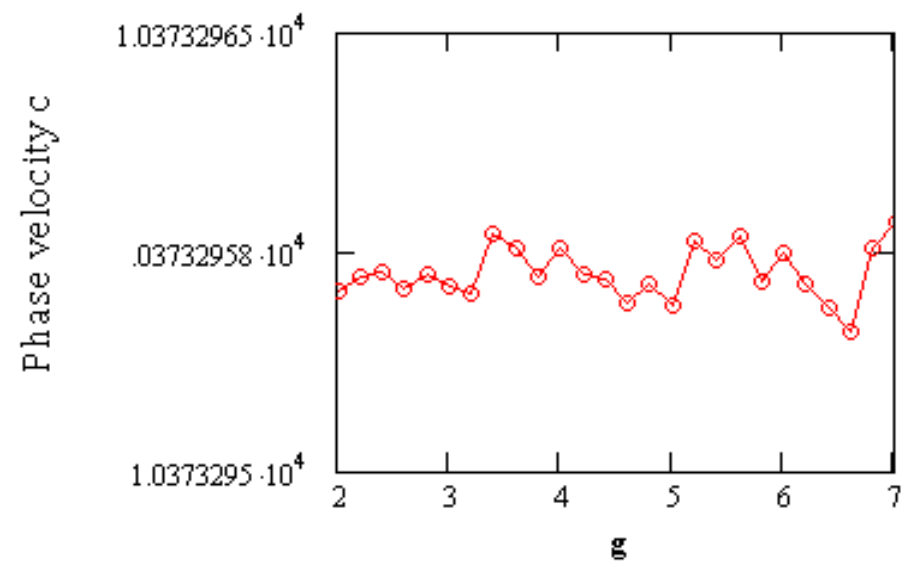

Figure 7. Phase velocity c for Rayleigh waves with respect to gravity $\mathrm{g}$.

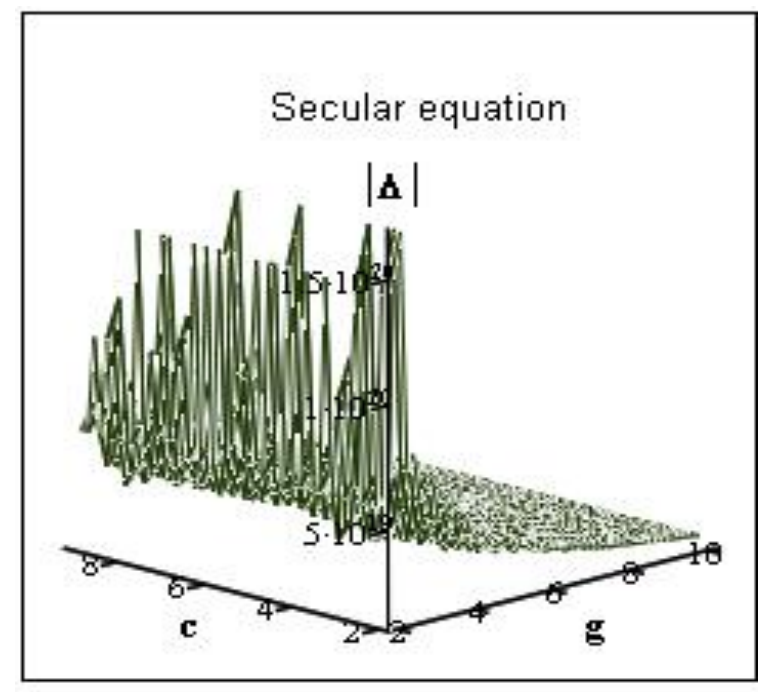

Figure 8. Variation of the secular equation for Rayleigh waves with varies values of $g$ and $c$.

wave speed and gravity field which it increases with increasing of depth, while it decreases with increasing of wave speed and gravity field, respectively. At a given instant, the velocity of Love wave is finite, which is due to the effect of gravity. Figure 11 shows that the variation of phase velocity of Love wave under the effects of gravity. The value of the phase velocity has an oscillatory behavior with gravity in the whole range of the depth, while it decreases with increasing of gravity field as well it increases with increasing of the value of depth.

Finally, Figures 12 and 13 show the variation of Love wave velocity, attenuation coefficient and secular equation with respect to depth $\mathrm{H}$ and phase velocity $\mathrm{C}$ which they increase with the increasing of wave speed and depth. 


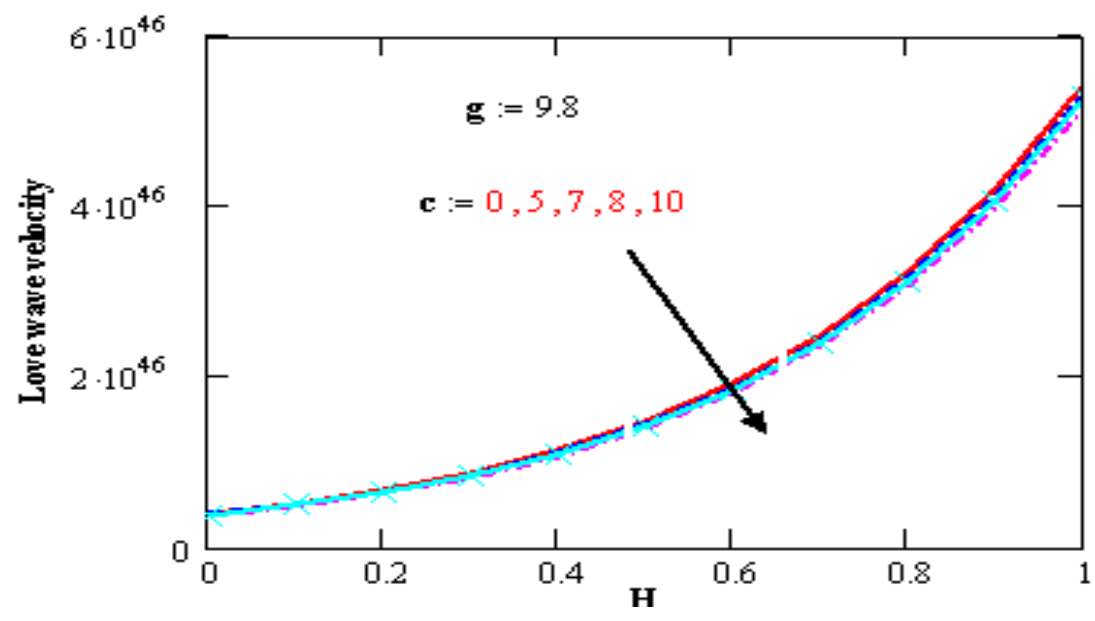

Figure 9. Variation of Love wave with varies values of $c$ with respect to the depth $\mathrm{H}$.

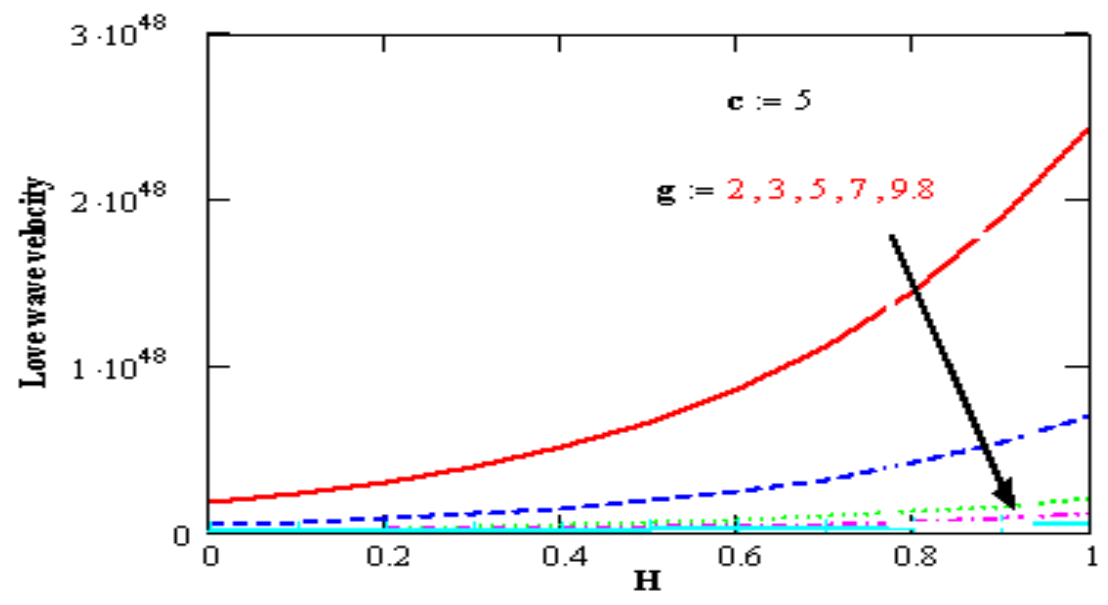

Figure 10. Variation of Love wave with varies values of $g$ with respect to the depth $\mathrm{H}$.

\section{Conclusion}

Due to the complicated nature of the governing equations of the elasticity fiber-reinforced theory, the work done in this field is unfortunately limited in number. The method used in this study provides a quite successful in dealing with such problems. This method gives exact solutions in the elastic medium without any assumed restrictions on the actual physical quantities that appear in the governing equations of the problem considered. Important phenomena are observed in all these computations:

1. It was found that for large values of time they give close results. The solutions obtained in the context of elasticity theory, however, exhibit the behavior of speeds of wave propagation.

2. By comparing Figures 1 to 12 , it was found that the wave velocity has the same behavior in both media. But with the passage of time and gravity, numerical values of wave velocity in the elastic medium are large in comparison due to the influences of gravity.

3. Special cases are considered as Rayleigh waves, Love wave and surface waves in anisotropic elastic medium, as well in the isotropic case.

4. The results presented in this paper should prove useful for researchers in material science, designers of new materials.

5. Study of the phenomenon of relaxation time and gravity is also used to improve the conditions of oil extractions.

Finally, if the gravity field is neglected, the relevant results obtained are deduced to the results obtained by Sengupta and Nath (2001). 

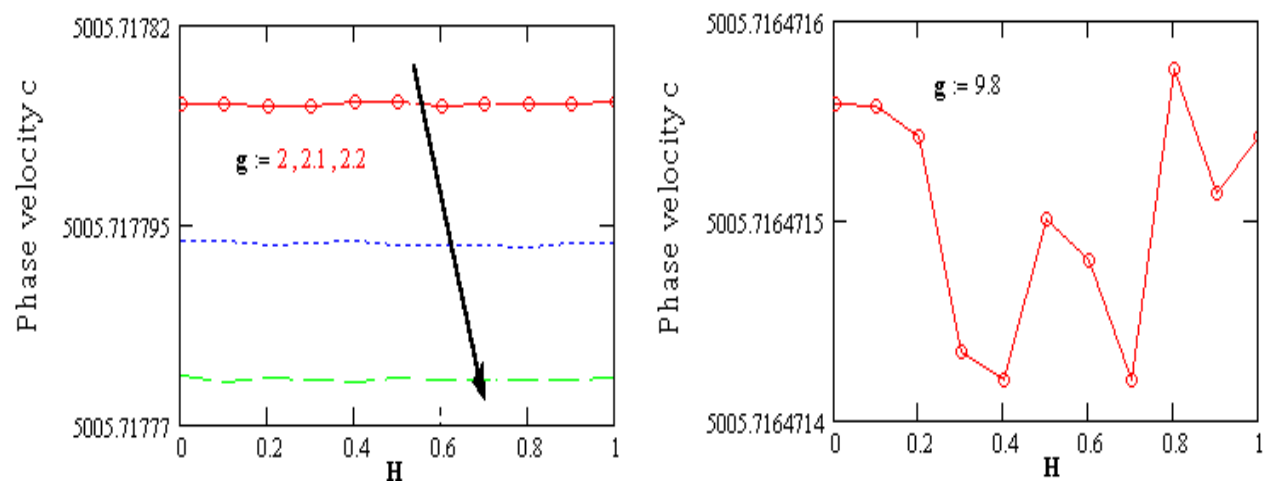

Figure 11. Variation of phase velocity with varies values of $g$ with respect to the depth $\mathrm{H}$.

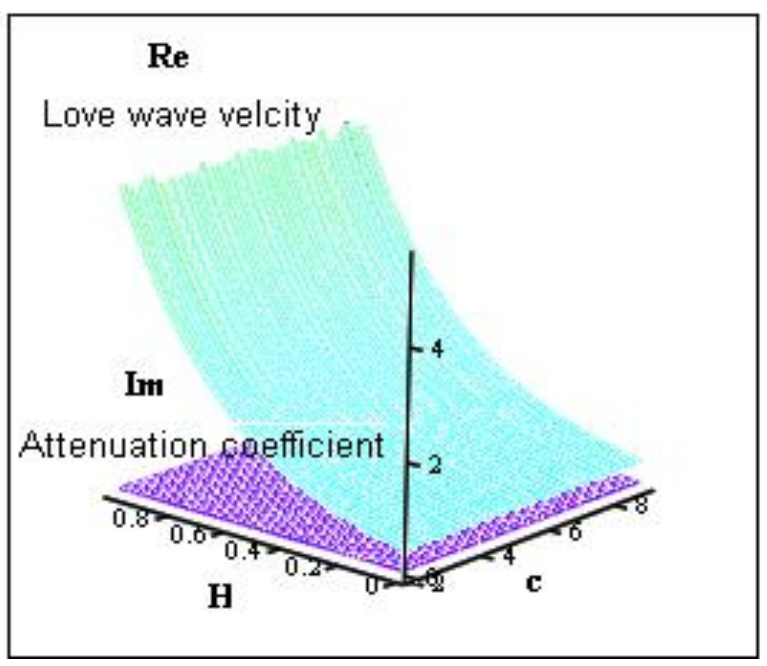

Figure 12. Variation of Love wave velocity and attenuation coefficient with varies values of $\mathrm{H}$ and $\mathrm{c}$.

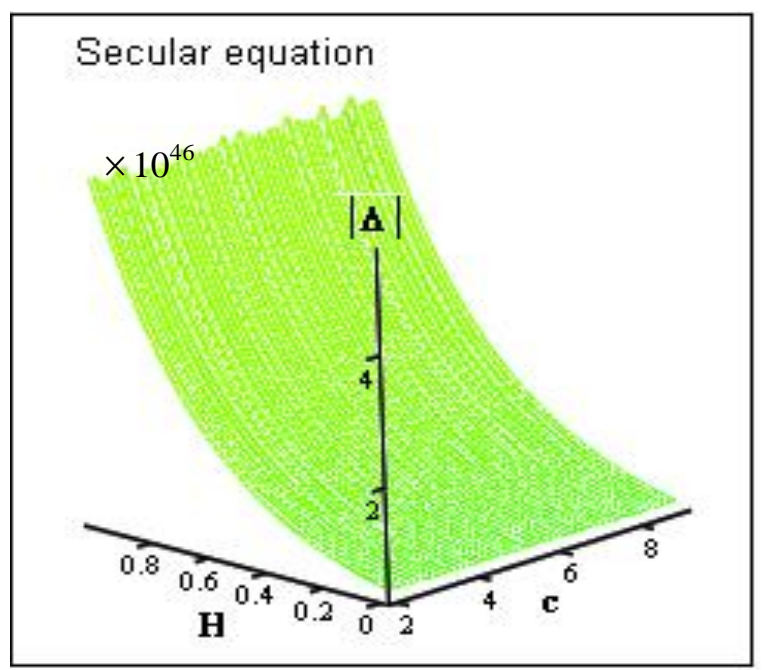

Figure 13. Variation of the secular equation for Love wave with varies values of $\mathrm{H}$ and $\mathrm{c}$.

\section{REFERENCES}

Abd-Alla AM, Ahmed SM (1999). Propagation of Love waves in a nonhomogeneous orthotropic elastic layer under initial stress overlying semi-infinite medium. Appl. Math. Comput. 106:265-275.

Abd-Alla AM (1999). Propagation of Rayleigh waves in an elastic halfspace of orthotropic material, Appl. Math. Comput. 99:61-69.

Abd-Alla AM, Abo-Dahab SM, Al-Thamali TA (2012). Propagation of Rayleigh waves in a rotating orthotropic material elastic half-space under initial stress and gravity. J. Mech. Sci. Technol. 26:2815-2823.

Abd-Alla AM, Abd-Alla AN, Zeidan NA (2000). Thermal stresses in a non-homogeneous orthotropic elastic multilayered cylinder. J. Therm. Stresses 23:313-428.

Abd-Alla AM, Abo-Dahab SM (2012). Effect of rotation and initial stress on an infinite generalized magneto-thermoelastic diffusion body with a spherical cavity. J. Therm. Stresses 35:892-912.

Abd-Alla AM, Abo-Dahab SM, Hammad HAH (2011). Propagation of Rayleigh waves in generalized magneto-thermoelastic orthotropic material under initial stress and gravity field. Appl. Math. Model 35:2981-3000.

Abd-Alla AM, Ahmed SM (2003). Stoneley and Rayleigh waves in a non-homogeneous orthotropic elastic medium under the influence of gravity. Appl. Math. Comput. 135:187-200.

Abd-Alla AM, Hammad HAH, Abo-Dahab SM (2004). Rayleigh waves in a magnetoelastic half-space of orthotropic material under influence of initial stress and gravity field. Appl. Math. Comput. 154:583-597.

Abd-Alla AM, SM Ahmed (1996). Rayleigh waves in an orthotropic thermoelastic medium under gravity field and initial stress. Earth Moon Planets 75:185-197.

Abouelregal AE, Abo-Dahab SM (2012). Dual phase lag model on magneto-thermoelasticity infinite non-homogeneous solid having a spherical cavity. J. Therm. Stresses 35:820-841.

Baljeet S (2005). Wave propagation in thermally conducting linear fibrereinforced composite materials. Arch. Appl. Mech. 75:513-520.

Baljeet S (2007). Wave propagation in an incompressible transversely isotropic fibre-reinforced elastic media. Arc. Appl. Mech. 77:253-258.

Chattopadhyay A, Venkateswarlu RLK, Saha S (2002). Reflection of quasi-P and quasi-SV waves at the free and rigid boundaries of a fibre-reinforced medium. Sãdhanã 27:613-630.

Dai HL, Wang X (2006). nStress wave propagation in piezoelectric fiber reinforced laminated composites subjected to thermal shock. Compos. Struct. 74:51-62.

Elnaggar AM, Abd-Alla AM (1989). Rayleigh waves in magneto-thermomicroelastic half-space under initial stress. Earth Moon Planets. 45:175-185.

Espinosa HD, Dwivedi S, Lu HC (2000). Modeling impact induced delamination of woven fiber reinforced composites with contact/cohesive laws. Comput. Methods Appl. Mech. Eng. 183:259290.

Fu YB, Zhang YT (2006). Continuum-mechanical modelling of kinkband formation in fibre-reinforced composites. Int. J. Solids 
Struct. 43:3306-3323.

Huang W, Rokhlin SI, Wang YJ (1995). Effect of fibre-matrix interphase on wave propagation along, and scattering from, multilayered fibres in composites. Transfer matrix approach. Ultrasonics 33:365-375.

Rogerson GA (1992). Enetration of impact waves in a six-ply fibre composite laminate. J. Sound Vib. 158:105-120.

Sang-Ho R, Jeong-Ki L, Jung-Ju L (2007). The group velocity variation of Lamb wave in fiber-reinforced composite plate. Ultrasonics 47:5563.

Sapan KS, Ranjan C (2011). Surface wave propagation in fiberreinforced anisotropic elastic layer between liquid saturated porous half space and uniform liquid layer. Acta Geophys. 59:470-482.

Sengupta PR, Nath S (2001). Surface waves in fibre-reinforced anisotropic elastic media. Sãdhanã 26:363-370.

Singh B, Singh SJ (2004). Reflection of plane waves at the free surface of a fibre-reinforced elastic half space. Sãdhanã 29:249-257.

Tadashi $O$ (2000). The propagation of Rayleigh waves along an obliquely cut surface in a directional fiber-reinforced composite. Compos. Sci. Technol. 60:2191-2196.
Weitsman $Y$ (1972). On wave propagation and energy scattering in materials reinforced by inextensible fibers. Int. J. Solids Struct. 8:627650.

Weitsman Y, Benveniste $Y$ (1974). On wave propagation in materials reinforced with bi-directional fibers. J. Sound Vib. 34:179-198.

Weitsrian $Y$ (1992). On the reflection of harmonic waves in fiberreinforced materials. J. Sound Vib. 26:73-89.

Wu TT, Chai JF (1994). Propagation of surface waves in anisotropic solids, theoretical calculation and experiment. Ultrasonics 32:21-29.

Wu TT, Liu YH (1999). On the measurement of anisotropic elastic constants of fiber-reinforced composite plate using ultrasonic bulk wave and laser generated Lamb wave. Ultrasonics 37:405-412. 